\title{
ON THE STRUCTURE OF ALGEBRAS AND HOMOMORPHISMS ${ }^{1}$
}

\author{
C. B. BELL
}

Introduction. The subject matter of this paper belongs to the general theory of sets. The objectives here are the examinations of the structure of classes of sets which are closed under various finite and transfinite set operations and those transformations which preserve these operations. The major results of this paper are partial extensions of (a) the well known theory of $\sigma$-algebras (e.g. [4]), (b) the work of E. Marczewski [1] on isomorphisms, (c) the work of R. Sikorski [2] on $\sigma$-homomorphisms and (d) the work of A. Tarski [3] on fields of sets and set functions. Three classes of sets-the algebra, $m$-algebra and total algebra-as well as four transformations-the $m$-homomorphism, weak isomorphism, $m$-isomorphism, and total isomorphism-are studied.

A class $\mathcal{K}$ of sets is an algebra if it is closed under finite set operations, i.e. addition of two sets and complementation. Analogically $\mathscr{K}$ is an $m$-algebra if $\nVdash$ is closed under $m$-operations, i.e. complementations and addition of not more than $m$ sets, where $m$ is an arbitrary fixed cardinal number. $\mathcal{K}$ is a total algebra if it is closed under all operations, i.e. complementation and arbitrary addition.

Two classes of sets, $\mathcal{K}$ and $\mathscr{L}$, are weakly isomorphic if $\mathcal{K}$ and $\mathscr{L}$ considered as partially ordered by the relation of proper inclusion are similar. $\mathcal{K}$ and $\mathscr{L}$ are $m$-isomorphic if they have the same properties from the point of view of $m$-operations; and, finally, $\mathfrak{K}$ and $\mathfrak{L}$ are totally isomorphic if they have the same properties from the point of view of all operations on sets.

The most important structure theorems concern the $m$-operations for $m \geqq \boldsymbol{\aleph}_{0}$. The formulation of the corresponding finite and total structure theorems follow easily from the $m$-theorems and will be omitted except in special cases.

The work is divided into two parts: (1) algebras of sets and (2) homomorphisms. In (1) the existence, composition and construction are treated; and, further, the relation between algebras and the "natural set units" is developed. In (2) are discussed some properties of $m$-additive, complementative transformations. Homomorphisms

Received by the editors November 9,1954 .

${ }_{1}^{1}$ The results presented here are a portion of the results of a research project which was financed in part by the Office of Naval Research. 
are characterized in terms of the "set units"; and several sets of equivalent conditions for homomorphisms and isomorphisms are given.

Terminology and notation. A space is understood to be an arbitrary nonvoid set. The letters $X$ and $Y$ will denote arbitrary spaces. $\varnothing$ will denote the empty set of any space. $\odot(X)$ denotes the class of all subsets of $X$. For each set $A \subset X, A^{0}$ denotes $X-A$ and $A^{1}$ denotes $A$.

Each class $\mathfrak{K}$ of subsets of $X$ will be considered indexed, i.e. can be represented in the form $\mathscr{K}=\left\{A_{v}\right\}_{v \in \widehat{K}}$. If $m$ is an arbitrary cardinal number, a class $\mathscr{K}$ of subsets of $X$ is called an $m$-class of subsets of $X$ whenever the cardinality, $\overline{\mathcal{K}}$, of $\mathcal{K}$ is such that $\overline{\mathcal{K}} \leqq m$.

A class $\mathscr{K}=\left\{A_{v}\right\}_{v \in \widehat{K}}$ of subsets of $X$ is called (1) complementative, (2) subtractive, (3) $m$-additive and (4) $m$-multiplicative, respectively, when for every $m$-class $\mathscr{T}=\left\{A_{v}\right\}_{v \in \widehat{\mathscr{N}}} \subset \mathscr{K}$, (1) $X-A_{v_{1}}=A_{v_{1}}^{0} \in \Re$, (2) $A_{v_{1}}-A_{v_{2}} \in \mathcal{K}$, (3) $\bigcup_{v \in \widehat{\mathscr{N}}} A_{v} \in \mathcal{K}$ and (4) $\bigcap_{v \in \widehat{\mathscr{N}}} A_{v} \in \mathcal{K}$. If $\mathcal{K}$ is $m$-additive [ $m$-multiplicative] for all finite $m, \mathfrak{K}$ is said to be additive [multiplicative]; if $\mathscr{K}$ is $m$-additive [ $m$-multiplicative] for all $m, \mathscr{K}$ is said to be totally additive [totally multiplicative]. It is easily seen that $\mathscr{K}$ is additive [multiplicative] if and only if $\mathscr{K}$ is $m$-additive [ $m$-multiplicative] for some $m \geqq 2$; totally additive [totally multiplicative], if and only if $\bar{\rho}(X)$-additive $[\bar{\rho}(X)$-multiplicative]. Further, one finds that each complementative, $m$-additive [ $m$-multiplicative] class is also $m$-multiplicative [ $m$-additive] for each $m$; and finally, that each complementative, multiplicative class is subtractive.

A mapping $\phi$ with domain $\mathcal{K}$ and range $\mathscr{L}$ is called a transformation of $\mathscr{K}$ onto $\mathcal{L}$. A transformation, $\phi$, of a class $\mathscr{K}$ (of subsets of $X$ ) onto a class $\mathscr{L}$ (of subsets of $Y$ ) is said to be complementative if for each $A, X-A=A^{0} \in \mathcal{K}, \phi(X-A)=Y-\phi(A)$, i.e. $\phi\left(A^{i}\right)=[\phi(A)]^{i}$ for $i=0,1$. Similarly, one defines subtractivity, $m$-additivity and $m$ multiplicativity for transformations. Finally, $\phi$ is inclusive if for each $A, B \in \mathcal{K}$ such that $A \subset B, \phi(A) \subset \phi(B)$.

\section{Algebras of Sets}

1.1. $m$-algebras. An $m$-algebra of subsets of $X$ is an $m$-additive, complementative class of subsets of $X$, an algebra is an $m$-algebra for all finite $m$; and a total algebra is an $m$-algebra for all $m$. [The usual $\sigma$-algebra is an $\boldsymbol{\aleph}_{0}$-algebra in the present notation.]

Since each $\bar{P}(X)$-algebra is a total algebra and each 2-algebra is an algebra, one need consider primarily $m$-algebras for $m \geqq \boldsymbol{\aleph}_{0}$ in order to grasp the general theory.

From the definitions it follows easily that each $m$-algebra is $m$ multiplicative, subtractive and contains $\varnothing$ and $X$. Further, to each 
class $\nVdash$ there is associated a unique $m$-algebra in the following manner.

TheOREM 1. If $\mathcal{K}$ is any class of subsets of $X$, then there exists a unique $m$-algebra $\mathcal{F}_{m}$, such that $\Re \subset \mathcal{F}_{m}$ and such that if $\mathcal{F}$ is any other m-algebra containing $\mathfrak{K}$, then $\mathcal{F}_{m} \subset \mathcal{F}$.

Proof. $P(X)$ is an $m$-algebra and, hence, there exists at least one $m$-algebra containing $\varkappa$. Furthermore, the intersection of an arbitrary family of $m$-algebras is again an $m$-algebra.

The intersection of all $m$-algebras containing $\mathscr{K}$ is, then, found to be the desired $m$-algebra $\mathcal{F}_{m}$.

The $m$-algebra $\mathcal{F}_{m}$, the least $m$-algebra containing $\mathscr{K}$, is called the $m$-algebra generated by $\mathcal{K}$; it will be denoted by $\mathcal{F}_{m}(\mathcal{K})$. Similarly, $\mathcal{F}(\mathcal{K})$ and $\mathcal{J}(\mathfrak{K})$ will denote, respectively, the least algebra and least total algebra containing $\mathscr{K}$.

Since each $m$-algebra is the least $m$-algebra containing some class of sets, the discussions of $m$-algebras can be limited to discussions of least $m$-algebras without loss of generality.

THEOREM 2. If $\mathfrak{K}$ is any class of subsets of $X$ and $A$ is any set of $\mathcal{F}_{m}(\mathcal{K})$, then there exists an $m$-subclass $\mathfrak{N}$ of $\mathscr{K}$ such that $A \in \mathcal{F}_{m}(\mathfrak{T})$; or equivalently $\mathcal{F}_{m}(\mathfrak{K})=U_{\mathfrak{T}} \subset \mathfrak{K}, \overline{\mathfrak{T}}=m \mathcal{F}_{m}(\mathfrak{T})$.

Proof. The union of all those $m$-subalgebras of $F_{m}(\mathcal{K})$ which are generated by some $m$-subclass of $\mathcal{K}$ is an $m$-algebra containing $\mathcal{K}$ and contained in $\mathcal{F}_{m}(\mathcal{K})$; it is therefore identical with $\mathcal{F}_{m}(\mathcal{K})$.

Theorem 2 above is essentially the classical composition theorem for $\sigma$-algebras. A more thorough understanding of the composition of $m$-algebras can be obtained if one studies the "natural set units" of $m$-algebras treated in the next section.

1.2. Constituents and atoms. If $\Re=\left\{A_{v}\right\}_{v \in \widehat{K}}$ is an arbitrary class of subsets of $X$, then each set of the form $\bigcap_{v \in \widehat{\Re}} A_{v}^{i_{v}}$, where $i_{v}=0$ or 1 for all $v$ and $\mathscr{N}=\left\{A_{v}\right\}_{v \in \widehat{\mathscr{N}}}$ is an $m$-subclass of $\mathscr{K}$, is called an $m$ constituent of $\mathscr{K}$; each $m$-constituent where $m$ is finite is called simply a constituent of $\mathscr{K}$; and each $\bigcap_{v \in \widehat{K}} A_{v}^{\xi_{v}}$ is a total constituent of $\mathscr{K}$. Each nonempty total constituent of $\mathscr{K}$ is called an atom of $\mathscr{K} \cdot \mathcal{S}(\mathcal{K})$ will denote the class of all atoms of $\mathcal{K}$.

For algebras it has been proved [1] that:

THeOREM 3. If $\mathfrak{K}$ is any class of sets, then $\mathcal{F}(\mathfrak{K})$ is the class of all finite unions of constituents of $\mathcal{K}$.

For total algebras an even stronger result is available. In fact 
Marczewski [1] has essentially proved the following theorem.

THEOREM 4. If $\mathcal{G}$ and $\mathfrak{K}$ are classes of subsets of $X$, then $\mathcal{G}=\mathcal{G}(\mathfrak{K})$, i.e. $\mathcal{G}$ is the class of all atoms of $K$, if and only if (a) the union of the elements of $\mathcal{G}$ is $X$; (b) the elements of $\mathcal{G}$ are mutually disjoint; (c) $\mathfrak{J}(\mathfrak{K})$ is the class of all unions of elements of $\mathcal{G}$; and (d) each element of $\mathfrak{J}(\mathfrak{K})$ can be uniquely represented as a union of elements of $\mathrm{G}$.

On the basis of the preceding two theorems one might be lead to believe that each $m$-algebra can be represented as the class of all $m$-unions of $m$-constituents. This result is unfortunately not true. The Borel sets in the unit interval provide a classic counterexample to the $m$-analog of Theorems 3 and 4 .

Let $X$ be the half open unit interval $[0,1]$. For $k=1,2, \cdots$, let $D_{k}$ be the set of all points of $X$, whose dyadic expansions $\left(\left\{i_{1}, i_{2}, \cdots\right\}\right.$ where $i_{k}=0$ or 1$)$ have 1 as the $k$ th digit. Let $\nVdash=\left\{D_{1}, D_{2}, \cdots\right.$, $\left.D_{n}, \cdots\right\} . F_{\aleph_{0}}(\mathcal{K})=B$, the class of Borel sets of the unit interval. The $\aleph_{0}$-constituents of $\mathcal{K}$ are the single points and finite unions of some special subintervals. It is well known that $\mathcal{F}_{\aleph_{0}}(\mathscr{K})=\mathscr{B}$ is most definitely neither the class of all $\aleph_{0}$-unions nor the class of all unions of the intervals and points mentioned above.

The strongest known theorem concerning the relationship between $m$-constituents and $m$-algebras is the following weak result.

THEOREM 5. If $\mathcal{K}$ is any class of sets, then $\mathfrak{F}_{m}(\mathcal{K})$ contains the class, $\mathfrak{T}_{m}$, of all $m$-unions of $m$-constituents of $\mathcal{K}$, and $\mathcal{F}_{m}(\mathfrak{K})$ is contained in the class $\mathfrak{J}_{m}$ of all unions of $m$-constituents of $\mathfrak{K}$; but there exists at least one class of sets $\mathfrak{K}_{1}$ and one infinite cardinal $m_{1}$ such that $\mathfrak{T}_{m_{1}} \neq \mathcal{F}_{m_{1}}\left(\mathcal{K}_{1}\right)$ $\neq \mathfrak{J}_{m_{1}}$.

1.3. Construction of $m$-algebras. Although a complete characterization of $m$-algebras in terms of $m$-constituents is not known, it is possible to formulate a transfinite construction process for least $m$ algebras. One such construction is described below.

Let $\mathcal{K}$ be an arbitrary class of subsets of $X$. Since $\mathcal{F}_{m}(\mathcal{K})$ $=\mathcal{F}_{m}(\mathfrak{K} \cup\{X\})$, one may assume without loss of generality that $X \in \mathcal{K}$. For each class $\mathfrak{D}$ of subsets of $X$, let $(D)_{m}$ be defined to be the class of all $m$-unions of differences of elements of $\mathscr{D}$. Let $\mathfrak{K}_{0}=\mathfrak{K}$ and $\mathfrak{K}_{\beta}=\left(\bigcup_{\alpha<\beta} \mathfrak{K}_{\alpha}\right)_{m}$ for each ordinal $\beta$. Now $\mathfrak{K}_{0} \subset \mathcal{F}_{m}(\mathfrak{K})$. Further, if $\mathfrak{K}_{\beta} \subset \mathcal{F}_{m}(\mathfrak{K})$ for all $\beta<\gamma$, then $\bigcup_{\beta<\gamma} \mathfrak{K}_{\beta} \subset \mathcal{F}_{m}(\mathfrak{K})$ and $\mathfrak{K}_{\gamma}=\left(\bigcup_{\beta<\gamma} \mathfrak{K}_{\beta}\right)_{m}$ $\subset \mathcal{F}_{m}(\mathscr{K})$. The use of the principle of transfinite induction is, then, sufficient to establish the fact that $\mathfrak{K}_{\alpha} \subset \mathcal{F}_{m}(\mathfrak{K})$ for all ordinals $\alpha$.

If $\Omega(m)$ is the first ordinal such that $\bar{\Omega}(m)>m$ and $\mathcal{F}_{m}=U_{\alpha<\Omega(m)} \mathcal{K}_{\alpha}$, the preceding paragraphs guarantee that $\mathfrak{K}_{\mathcal{C}} \subset \mathcal{F}_{m} \subset \mathcal{F}_{m}(\mathfrak{K})$. 
Further, if $A, B \in \mathcal{F}_{m}$, there exist ordinals $\alpha, \beta$ and $\gamma$ such that $A \in \mathcal{K}_{\alpha}, B \in \mathcal{K}_{\beta}$ and $\alpha, \beta<\gamma<\Omega(m)$. Consequently, $A-B \in \mathcal{K}_{\gamma} \subset \mathcal{F}_{m}$. In particular $X-B \in \mathcal{F}_{m}$ for all $B \in \mathcal{F}_{m}$. $\mathcal{F}_{m}$ is, therefore, complementative.

If now $\mathscr{N}=\left\{B_{v}\right\}_{v \in \widehat{N}}$ is an arbitrary $m$-subclass of $\mathcal{F}_{m}$, there exists for each $v \in \widehat{\mathscr{N}}$ an ordinal $\alpha(v)$ such that $B_{v} \in \mathcal{K}_{\alpha(v)}$. If $\sigma=\sum_{v \in \widehat{\mathscr{N}}} \alpha(v)$, $\bar{\sigma}=\sum_{v \in \widehat{\mathscr{N}}} \bar{\alpha}(v) \leqq m \cdot m=m ; \sigma>\alpha(v)$ for all $v \in \widehat{\mathscr{N}}$ and $\sigma<\Omega(m)$. Hence $U_{v \in \widehat{\mathscr{N}}} B_{v} \in \mathcal{K}_{\sigma} \subset \mathcal{F}_{m}$ and $\mathcal{F}_{m}$ is $m$-additive.

These considerations establish the following theorem.

TheOREM 6. If $\mathcal{K}_{0}=\mathfrak{K}$ is a class of subsets of $X$ which contains $X$; $(\mathfrak{L})_{m}$ is the class of all $m$-unions of differences of sets of $\mathfrak{L}$ for each $\mathcal{L} \mathcal{P}(X) ; \Omega(m)$ is the first ordinal such that $\bar{\Omega}(m)>m ;$ and $\mathcal{K}_{\beta}$ $=\left(\bigcup_{\alpha<\beta} \mathcal{K}_{\alpha}\right)_{m}$ for all $\beta$, then

$$
\mathcal{F}_{m}(\mathfrak{K})=\underset{\alpha<\Omega(m)}{\mathfrak{K}_{\alpha}}
$$

An immediate corollary to the construction theorem is the hierarchy theorem below.

THEOREM 7. If $\mathscr{K}$ is any class of subsets of $X$ and $m$ and $n$ are two infinite cardinals such that $n<m$, then

$$
\mathfrak{K} \subset \mathcal{F}(\mathfrak{K}) \subset F_{n}(\mathfrak{K}) \subset \mathscr{F}_{m}(\mathfrak{K}) \subset \mathfrak{I}(\mathfrak{K}) \subset \mathcal{P}(X),
$$

where the first, second, third, fourth and fifth inclusions are, respectively, proper if and only if (1) $K$ is not an algebra; (2) $\bar{\kappa} \geqq \aleph_{0}$; (3) $\bar{\kappa}>n$, (4) $\bar{K}>m$; and (5) at least one atom of $\mathscr{K}$ contains more than one point of $X$.

\section{TRANSFORMATIONS}

2.1. Homomorphisms. Since an $m$-algebra is an $m$-additive, complementative class of sets, it is not unreasonable to expect that an $m$-additive, complementative transformation will preserve the algebraic properties of an $m$-algebra. This result is in fact true.

THEOREM 8. If $\phi$ is an $m$-additive, complementative transformation of an $m$-algebra $\mathfrak{T}$ of subsets of $X$ onto a class $\mathcal{L}$ of subsets of $Y$, then $\mathfrak{L}$ is an m-algebra. If, further, $\mathscr{K} \subset \mathfrak{T}$, then $\phi\left(\mathcal{F}_{m}(\mathfrak{K})\right)=\mathcal{F}_{m}(\phi(\mathfrak{K}))$.

Proof. To prove the theorem it is sufficient to prove the latter conclusion. To establish this result it is sufficient to prove that $\phi\left(\mathfrak{K}_{\beta}\right)$ $=(\phi(\mathcal{K}))_{\beta}$ for all $\beta<\Omega(m)$. The proof is by transfinite induction.

(a) $\phi\left(\mathcal{K}_{0}\right) \equiv \phi(\mathcal{K}) \equiv(\phi(\mathcal{K}))_{0}$.

(b) Assume that $\phi\left(\mathcal{K}_{\alpha}\right)=(\phi(\mathcal{K}))_{\alpha}$ for all $\alpha<\beta$.

(c) Since $\phi$ is $m$-additive and complementative on $m$-algebra $\mathfrak{N}$. 
it is also $m$-multiplicative and subtractive on $\mathfrak{T}$. These properties and assumption (b) guarantee that

$$
\begin{aligned}
\phi\left(\mathcal{K}_{\beta}\right) & =\phi\left(\left(\bigcup_{\alpha<\beta} \mathfrak{K}_{\alpha}\right)_{m}\right)=\left(\phi\left(\bigcup_{\alpha<\beta} \mathcal{K}_{\alpha}\right)\right)_{m}=\left(\bigcup_{\alpha<\beta} \phi\left(\mathcal{K}_{\alpha}\right)\right)_{m} \\
& =\left(\bigcup_{\alpha<\beta}(\phi(\mathcal{K}))_{\alpha}\right)_{m}=(\phi(\mathscr{K}))_{\beta}
\end{aligned}
$$

for all $\beta$ and in particular for all $\beta<\Omega(m)$. The theorem, then, is proved.

From the above theorem one is led in a natural manner to the following definition of an $m$-homomorphism.

A transformation $\phi$ of a class $\mathscr{K}$ of subsets of $X$ onto a class $\mathscr{L}$ of subsets of $Y$ is called an $m$-homomorphism of $\nVdash$ onto $\mathscr{L}$, if there exists an extension $\phi^{*}$, of $\phi$, which is an $m$-additive, complementative transformation of $\mathcal{F}_{m}(\mathscr{K})$ onto $\mathcal{F}_{m}(\mathfrak{L})$. Homomorphisms and total homomorphisms are defined analogously.

The elementary properties of $m$-homomorphisms are the usual ones. It is readily established that the extension $\phi^{*}$, of an $m$-homomorphism $\phi$, is $m$-multiplicative, subtractive and inclusive; and, further, that $\phi^{*}(X)=Y$ and $\phi^{*}(\varnothing)=\varnothing$.

The most important property of an $m$-homomorphism $\phi$ concerns the behavior of the $m$-constituents and their images under $\phi$. If $\phi$ is a transformation of $\mathcal{K}$ onto $\mathscr{L}$, and $\bigcap_{v \in \widehat{\mathscr{T}}} A_{v}^{\mathfrak{t}_{v}}$ is an $m$-constituent of $\mathscr{K}$, then $\bigcap_{v \in \widehat{\mathscr{N}}}\left[\phi\left(A_{v}\right)\right]^{i_{v}}$ is the corresponding $m$-constituent of $\mathscr{L}$ under $\phi$. In terms of these entities the $m$-homomorphism can be characterized as follows.

TheOREM 9. A transformation $\phi$ of $\mathcal{K}$ onto $\mathfrak{L}$ is an $m$-homomorphism of $\mathcal{K}$ onto $\mathcal{L}$ if and only if $(\delta m)$ : whenever an $m$-constituent of $\mathcal{K}$ is empty so is its corresponding m-constituent.

Proof (Necessity). If $\phi$ is an $m$-homomorphism and $\phi^{*}$ is its $m$-additive, complementative extension, then $\phi^{*}(\varnothing)=\varnothing$. Further, each $m$-constituent of $\mathscr{K}$ is an element of $\mathcal{F}_{m}(\mathfrak{K})$. Hence $(\delta m)$ holds.

(Sufficiency). Suppose condition $(\delta m)$ holds, i.e. if $\bigcap_{v \in \widehat{\mathscr{N}}} A_{v}^{\mathfrak{i}_{v}}=\varnothing$, then $U_{v \in \widehat{\mathscr{T}}}\left[\phi\left(A_{v}\right)\right]^{\mathfrak{v}_{v}}=\varnothing$ for all $m$-subclasses, $\mathscr{N}=\left\{A_{v}\right\}_{v \in \widehat{\mathscr{N}}}$, of $\mathscr{K}$. Let $A$ be an arbitrary element of $\mathcal{F}_{m}(\mathcal{K})$. Then there exists (Theorem 2) an $m$-subclass, $\mathscr{N}=\left\{A_{v}\right\}_{v \in \widehat{\mathscr{N}}}$, of $\Re$ such that $A \in \mathcal{F}_{m}(\mathfrak{N}) \subset \Im(\Re)$. But each set expressible in the form $\bigcap_{v \in \widehat{\mathscr{N}}} A_{v}^{t_{0}}$ is a total constituent of $\Re$ (as well as an $m$-constituent of $\mathscr{K}$ ). Therefore, $A$ can be represented (Theorem 4) uniquely, up to empty total constituents, as a 
sum of total constituents of $\Re$. Define

$$
\phi \Re(A)=\bigcup_{\bigcap_{v} \in \widehat{\mathscr{T}} A_{v}^{i_{v}} \subset_{A}}\left(\bigcap_{v \in \widehat{\mathscr{N}}}\left[\phi\left(A_{v}\right)\right]_{i_{\nu}}\right) .
$$

Since $(\delta m)$ and unique representation hold, $\phi_{\mathscr{T}}$ is well defined for all elements of $\mathfrak{J}(\mathscr{T})$ and, in particular, the elements of $\mathcal{F}_{m}(\mathscr{T})$.

Now let $\mathfrak{T}=\left\{A_{v}\right\}_{v \in \widehat{\mathscr{N}}}$ be an arbitrary $m$-subclass of $\mathfrak{K}$ such that $\mathscr{T}$ is a subclass of $\mathscr{T}$. It is seen that $\mathcal{F}_{m}(\mathfrak{T}) \supset \mathcal{F}_{m}(\mathfrak{T})$; in fact, each atom of $\mathscr{N}$ is uniquely representable as a sum of atoms of $\mathscr{T}$ (Theorem 4). Define $\phi \mathfrak{T I}(A)$ in the manner of the preceding paragraph. It is found, then, that since $Y$ is the union of all of the total constituents of $\phi(\mathfrak{T}-\mathfrak{T})$ (Theorem 4),

$$
\begin{aligned}
& \phi \mathfrak{N}(A)=\bigcap_{v \in \widehat{\mathscr{N}} A_{v}^{i_{v}} \subset A}\left(\bigcap_{v \in \widehat{\mathscr{N}}}\left[\phi\left(A_{v}\right)\right]^{i_{v}}\right) \\
& =\left(\underset{\bigcap_{v} \in \widehat{\mathscr{T}} A_{v}^{i_{v}} \subset A}{U}\left(\bigcap_{\widehat{v} \in \widehat{\mathscr{T}}}\left[\phi\left(A_{v}\right)\right]^{i_{v}}\right)\right)_{\bigcap} \\
& \left.\cdot\left(\bigcap_{v \in \widehat{\mathscr{N}}-\widehat{\mathscr{N}} A_{v}^{i_{v}} \subset C_{X}\left(\bigcap_{v} \in \widehat{\mathscr{N}}-\widehat{\mathscr{N}}\right.}\left[\phi\left(A_{v}\right)\right]^{i_{v}}\right)\right)
\end{aligned}
$$

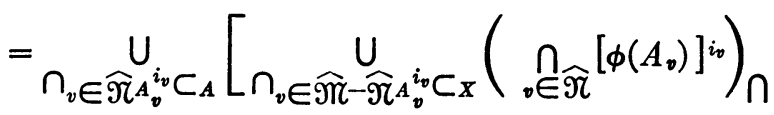

$$
\begin{aligned}
& \left.\cdot\left(\bigcap_{v} \in \widehat{\mathscr{N}}-\widehat{\mathscr{T}}\left[\phi\left(A_{v}\right)\right]^{i_{v}}\right)\right] \\
& =\underset{\bigcap_{v} \in \widehat{\mathscr{N}} A_{v}^{i_{v}} C_{A}}{U}\left(\bigcap_{v \in \widehat{\mathscr{N}}}\left[\phi\left(A_{v}\right)\right]^{i_{v}}\right)=\phi_{\mathscr{T}}(A) \text {. }
\end{aligned}
$$

Therefore, for any $m$-subclass $B$ of $\mathscr{K}$ such that $A \in \mathcal{F}_{m}(B), \phi_{\mathscr{N}}(A)$ $=\phi_{\mathscr{T} \cup B}(A)=\phi_{B}(A)$. [ $\mathfrak{N \cup} B$ is an $m$-subclass of $\mathscr{K}$ containing both $\Re$ and $B$.

Define now for each $A \in \mathcal{F}_{m}(\mathfrak{K}), \phi^{*}(A)=\phi_{\mathfrak{T C}}(A)$, where $\mathfrak{T C}$ is an arbitrary $m$-subclass of $\mathscr{K}$ such that $A \in \mathcal{F}_{m}(\mathscr{T})$. $\phi^{*}$ is well defined.

$\{A\}$ is an $m$-subclass of $\mathscr{K}$ such that $A \in \mathcal{F}_{m}(\{A\})$. Therefore, $\phi^{*}(A)=\phi_{\{A\}}(A)=\phi(A)$ for all $A \in \mathcal{K}$. Hence $\phi^{*}$ is an extension of $\phi$.

It will be sufficient to prove the complementativity and $m$-additivity of $\phi^{*}$, in order to complete the proof of the theorem.

(a) For any $m$-subclass, $\mathcal{U}$ of $\mathscr{K}, \phi \mathcal{U}(X)$ is the union of all total constituents of $\phi(\mathfrak{U}) \subset \mathfrak{L}$. Hence $\phi^{*}(X)=\phi_{\mathfrak{U}}(X)=Y$.

(b) For arbitrary $A \in \mathcal{F}_{m}(\mathcal{K})$, it is already known that there exists an $m$-subclass, $\mathcal{V}=\left\{A_{v}\right\}_{v \in \widehat{\mathcal{V}}}$, of $\mathscr{K}$ such that $A \in \mathcal{F}_{m}(\mathcal{V})$. Then, since 
representation in $J(V)$ is unique up to empty total constituents of $V$,

$$
\begin{aligned}
& \phi^{*}\left(A^{0}\right)=\phi^{*}(X-A)=\underset{\bigcap_{v} \in \widehat{\mathcal{V}} A_{v}^{i_{v}} \subset_{X-A}}{\bigcup}\left(\bigcap_{v} \in \mathcal{V}\left[\phi\left(A_{v}\right)\right]^{i_{v}}\right) \\
& =\cap_{v \in \widehat{\mathcal{V}} A_{v}^{i_{v}} \subset X}\left(\bigcup_{v \in \widehat{\mathcal{V}}}\left[\phi\left(A_{v}\right)\right]^{i_{v}}\right) \\
& \text { - } \bigcap_{v \in \widehat{\mathcal{U}} A_{v}^{i_{v}} \subset A}\left(\bigcap_{v \in \widehat{V}}\left[\phi\left(A_{v}\right)\right]^{i_{v}}\right) \\
& =\phi^{*}(X)-\phi^{*}(A)=Y-\phi^{*}(A)=\left[\phi^{*}(A)\right]^{0}
\end{aligned}
$$

and $\phi^{*}$ is complementative on $\Re$.

(c) Let $B=\left\{B_{s}\right\}_{s \in \widehat{B}}$ be an $m$-subclass of elements of $\mathcal{F}_{m}(\mathfrak{K})$. For each $s \in \widehat{\mathscr{B}}$, there exists an $m$-subclass, $\mathscr{T}_{s}=\left\{A_{v}\right\}_{v \in \widehat{\mathscr{T}}_{s}}$ of $\mathscr{K}$ such that $B \in \mathcal{F}_{m}\left(\mathfrak{M T}_{\mathrm{s}}\right)$.

$$
\mathscr{M}=\bigcup_{\bullet \in \widehat{\mathscr{B}}} \mathscr{M T}_{i}=\left\{A_{v}\right\}_{v} \in U_{s \in \widehat{\mathscr{B}}} \widehat{\mathscr{M}}_{i}=\widehat{\mathscr{M}}
$$

is also an $m$-subclass of $\mathfrak{K}$. Therefore,

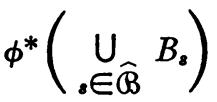

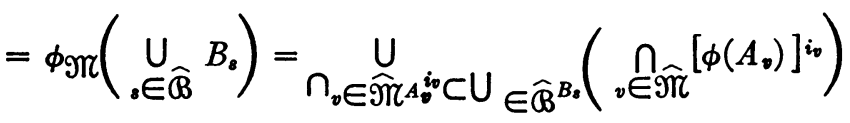

$$
\begin{aligned}
& =\bigcup_{s \in \widehat{\mathscr{B}}}\left(\bigcap_{v \in \widehat{\mathscr{M}}^{A_{v}}{ }_{v}^{i_{v}} \subset_{B_{s}}}\left(\bigcap_{v \in \widehat{\mathscr{M}}}\left[\phi\left(A_{v}\right)\right]^{i_{v}}\right)\right)=\bigcup_{s \in \widehat{\mathscr{B}}} \phi^{*}\left(B_{s}\right) .
\end{aligned}
$$

$\phi^{*}$ is, then, $m$-additive and the theorem is proved.

Similar theorems hold for homomorphisms and total homomorphisms. The other special homomorphisms of interest are the isomorphisms. These are treated in the next section.

2.2. Isomorphisms. A $1-1$ transformation $\phi$ of $\mathcal{K}$ onto $\mathscr{L}$ is said to be an $m$-isomorphism of $\mathcal{K}$ onto $\mathscr{L}$ if there exists an extension $\phi^{*}$, of $\phi$, which is a $1-1, m$-additive, complementative transformation of $\mathcal{F}_{m}(\mathfrak{K})$ onto $\mathcal{F}_{m}(\mathscr{L})$. Isomorphisms and total isomorphisms are analogously defined. A transformation $\phi$ of $\mathcal{K}$ onto $\mathscr{L}$ is said to be a weak isomorphism of $\Re$ onto $\mathcal{L}$ if $\phi$ is $1-1$ and both $\phi$ and $\phi^{-1}$, the inverse of $\phi$, are inclusive.

From the definitions it is readily seen that an $m$-isomorphism is simply a "two-way" $m$-homomorphism and has the corresponding properties. What cannot be readily seen is that a weak isomorphism, when its domain and range are $m$-algebras, is an $m$-isomorphism, i.e. 
the preservation of order (by inclusion) is sufficient to establish $m$-additivity, complementativity, etc. These results are summarized in Theorem 10.

THEOREM 10. The following four conditions are equivalent:

(1) $\phi$ is an $m$-isomorphism of $\mathcal{K}$ onto $\mathcal{L}$;

(2) $\phi$ satisfies $(\eta m)$ : corresponding m-constituents under $\phi$ are simultaneously empty or not;

(3) $\phi$ can be extended to a weak isomorphism of $\mathcal{F}_{m}(\mathcal{K})$ onto $\mathcal{F}_{m}(\mathcal{L})$; and

(4) $\phi$ is an $m$-homomorphism of $\mathcal{K}$ onto $\mathscr{L}$ and $\phi^{-1}$ is an $m$-homomorphism of $\mathscr{L}$ onto $\mathfrak{K}$.

Proof. The facts that (1) implies (3) and that (1), (2) and (4) are equivalent follow immediately from Theorem 9 and the definitions. To complete the proof it will be sufficient to prove that the extension $\phi^{*}$, of $\phi$, which is a weak isomorphism of $\mathcal{F}_{m}(\mathcal{K})$ onto $\mathcal{F}_{m}(\mathscr{L})$, is $m$-additive and complementative. [To simplify notation $\phi$ will be substituted for $\phi^{*}$ in the following proof.]

If $\mathscr{T}=\left\{A_{v}\right\}_{v \in \widehat{\mathscr{N}}}$ is any $m$-subclass of $\mathcal{F}_{m}(\mathscr{K})$, then since $\phi$ is inclusive, $\phi\left(A_{v}\right) \subset \phi\left(\bigcup_{v \in \widehat{\mathscr{N}}} A_{v}\right)$ for all $v \in \widehat{\mathscr{N}}$ and $\bigcup_{v \in \widehat{\mathscr{N}}} \phi\left(A_{v}\right) \subset \phi\left(\bigcup_{v \in \widehat{\mathscr{N}}} A_{v}\right)$. But $\phi^{-1}$ is also inclusive and, therefore,

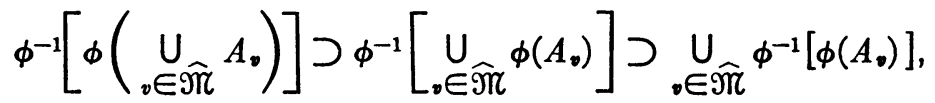

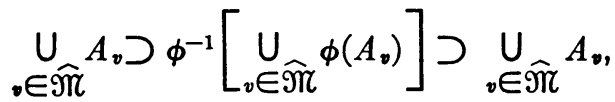

$$
\begin{aligned}
& \phi\left(\bigcup_{v \in \widehat{\mathscr{T}}} A_{v}\right) \supset \underset{v \in \widehat{\mathscr{M}}}{\bigcup_{\widehat{T}}} \phi\left(A_{v}\right) \supset \phi\left(\bigcup_{v \in \widehat{\mathscr{M}}} A_{v}\right) \text {. }
\end{aligned}
$$

So $\phi\left(\bigcup_{v} \in \widehat{\mathscr{N}} A_{v}\right)=\mathrm{U}_{v} \in \widehat{\mathscr{N}} \phi\left(A_{v}\right)$ and $\phi$ is $m$-additive.

Since $\phi$ and $\phi^{-1}$ are both inclusive, $\phi(X) \supset \phi(A)$ for all $A \in \mathcal{F}_{m}(\mathfrak{K})$ and $\phi^{-1}(Y) \supset \phi(B)$ for all $B \in \mathcal{F}_{m}(\mathscr{L})$. But $\phi\left(\mathcal{F}_{m}(\mathscr{K})\right)=\mathcal{F}_{m}(\mathscr{L})$ and $\phi^{-1}\left(\mathcal{F}_{m}(\mathcal{L})\right)=\mathcal{F}_{m}(\mathfrak{K})$. Consequently, $\phi(X) \supset Y, \phi^{-1}(Y) \supset X$, and $\phi(A)$ $=Y$ if and only if $A=X$. Further, $\phi(A)=\varnothing$ if and only if $A=\varnothing$.

If $B$ is any element of $\mathcal{F}_{m}(\mathcal{K})$, then $Y=\phi(X)=\phi\left(B^{0} \cup B\right)=\phi\left(B^{0}\right)$ $\cup_{\phi}(B)$. If $\phi\left(B^{0}\right) \cap \phi(B)=\varnothing$, then $\phi\left(B^{0}\right)=Y-\phi(B)=[\phi(B)]^{0}$ and $\phi$ would be complementative. If $C$ is such that $\phi(C)=\phi\left(B^{0}\right) \cap \phi(B)$, then $\phi(C) \subset \phi\left(B^{0}\right), \phi(B)$ and $C \subset B^{0}, B$. Therefore $C=\varnothing, \phi(C)=\varnothing$ and $\phi$ is complementative. The theorem is proved.

The theorem for total isomorphisms analogous to Theorem 10 can be obtained from Theorem 10 by putting $m=\bar{\Phi}(X)$. However, Theo- 
rem 5 enables one to establish an added equivalent condition for total isomorphisms.

THEOREM 11. The following five conditions are equivalent:

(1) $\phi$ is a total isomorphism of $\mathcal{K}$ onto $\mathcal{L} ;(2) \phi$ and $\phi^{-1}$ are both total homomorphisms; (3) $\phi$ can be extended to a weak isomorphism of $J(\mathfrak{K})$ onto $J(\mathscr{L}) ;(4) \phi$ satisfies condition $\left(\eta_{T}\right)$ : Corresponding total constituents are simultaneously void or not; and (5) $\phi$ induces and is induced by a 1-1 transformation of $\mathcal{G}(\mathcal{K})$ onto $\mathrm{G}(\mathfrak{L})$.

\section{BIBLIOGRAPHY}

1. E. Marczewski, On the isomorphism and the equivalence of classes and sequences of sets, Fund. Math. vol. 32 (1939) pp. 133-148.

2. R. Sikorski, On an analogy between measures and homomorphisms, Annales de la Société Polonaise de Mathématique vol. 23 (1950) pp. 1-20.

3. A. Tarski, Über additiv und multiplikativ Mengenkörper und Mengenfunktionen, Comptes Rendus des Séances de la Société des Sciences et des Lettres de Varsovie vol. 30 (1937) pp. 151-181.

4. P. Halmos, Measure theory, Van Nostrand, 1950, pp. 19-29.

5. C. Bell, Structures of measure spaces, Ph.D. thesis, University of Notre Dame, 1953.

UNIVERSITY OF NOTRE DAME AND

XAVIER UNIVERSITY OF LoUisiana 